\title{
3D Simulation Studies of the Two-Stream Instability in Intense Particle Beams Based on the Vlasov-Maxwell Equations *
}

\author{
Hong Qin, Ronald C. Davidson, Edward Startsev, and W. Wei-li Lee \\ Princeton Plasma Physics Laboratory, Princeton University, Princeton, NJ 08543
}

\begin{abstract}
Two-stream instabilities in intense charged particle beams, described self-consistently by the nonlinear Vlasov-Maxwell equations, are studied using a 3D multispecies perturbative particle simulation method. The newly developed beam equilibrium, stability, and transport (BEST) code is used to simulate the linear and nonlinear properties of the electron-proton (e-p) two-stream instability observed in the Proton Storage Ring (PSR) experiment for a long coasting beam. Simulations in a parameter regime characteristic of the PSR experiment show that the e-p instability has a dipole-mode structure, and that the instability threshold descreases with increasing fractional neutralization, and increases with increasing axial momentum spread of the beam particles. In the nonlinear phase, the simulations show that the instability first saturates at a relatively low level, and subsequently grows to a higher level.
\end{abstract}

\section{INTRODUCTION}

In periodic focusing accelerators and transport systems [1-4], when a second charge component is present, it has been recognized for many years, both in theoretical studies and in experimental observations [5-18], that the relative streaming motion of the high-intensity beam particles through a background charge species provides the free energy to drive the classical two-stream instability, appropriately modified to include the effects of dc space charge, relativistic kinematics, presence of a conducting wall, etc. A background population of electrons can result by secondary emission when energetic beam ions strike the chamber wall, or through ionization of background neutral gas by the beam ions. A well-documented example is the electron-proton (e-p) instability observed in the Proton Storage Ring [12-14], although a similar instability also exists for other ion species, including (for example) electronion interactions in electron storage rings [15-18].

At the high beam currents and charge densities of practical interest, it is increasingly important to develop an improved theoretical understanding of the influence of the intense self fields using a kinetic model based on the nonlinear Vlasov-Maxwell equations [1, 19-22]. Recently, the $\delta \mathrm{f}$ formalism, a low-noise, nonlinear perturbative particle simulation technique for solving the Vlasov-Maxwell

* This research was supported by the Department of Energy and the Spallation Neutron Source (SNS) project. We thank R. Macek, T.-S. Wang, P. Channell, K. Harkay, and M. Blaskiewicz for productive discussions and comments. equations, has been developed for intense beam applications $[23,24]$. The 3D multispecies nonlinear $\delta f$ formalism has been implemented in the newly developed Best Equilibrium Stability and Transport (BEST) code [25], which has been applied to a wide range of important collective processes in intense beams [25, 26]. In this paper, we study the electron-proton two-stream instability numerically using the nonlinear $\delta f$ method, with particular emphasis on the parameter regime characteristic of the Proton Storage Ring (PSR) experiment [12-14] for a long coasting beam. Following a brief description of the nonlinear $\delta f$ method in Sec. 2, we present the simulation results in Sec. 3. In particular, the dependence of the instability growth rate on beam density, fractional charge neutralization, and longitudinal momentum spread is investigated in detail. The nonlinear phase of the instability is studied as well.

\section{NONLINEAR $\delta$ F FORMALISM FOR VLASOV-MAXWELL SYSTEM}

The theoretical model employed here is based on the nonlinear Vlasov-Maxwell equations. We consider a thin, continuous, high-intensity ion beam $(j=b)$, with characteristic radius $r_{b}$ propagating in the $z$-direction through background electrons $(j=e)$, with each component described by a distribution function $f_{j}(\mathbf{x}, \mathbf{p}, t)[5,20,22]$. The charge components $(j=b, e)$ propagate in the $z$ direction with characteristic axial momentum $\gamma_{j} m_{j} \beta_{j} c$, where $V_{j}=\beta_{j} c$ is the average directed axial velocity, $\gamma_{j}=\left(1-\beta_{j}^{2}\right)^{-1 / 2}$ is the relativistic mass factor, $e_{j}$ and $m_{j}$ are the charge and rest mass, respectively, of a $j$ 'th species particle, and $c$ is the speed of light in vacuo. While the nonlinear $\delta f$ formalism outlined here is readily adapted to the case of a periodic applied focusing field [27], for present purpose we make use of a smooth-focusing model in which the applied focusing force is described by $\mathbf{F}_{j}^{f o c}=$ $-\gamma_{j} m_{j} \omega_{\beta j}^{2} \mathbf{x}_{\perp}$, where $\mathbf{x}_{\perp}=x \hat{\mathbf{e}}_{x}+y \hat{\mathbf{e}}_{y}$ is the transverse displacement from the beam axis, and $\omega_{\beta j}=$ const is the effective applied betatron frequency for transverse oscillations. Furthermore, in a frame of reference moving with axial velocity $\beta_{j} c$, the motion of a $j$ 'th species particle is assumed to be nonrelativistic. The space-charge intensity is allowed to be arbitrarily large, subject only to transverse confinement of the beam ions by the applied focusing force, and the background electrons are confined in the transverse plane by the space-charge potential $\phi(\mathbf{x}, t)$ produced by the excess ion charge. In the electrostatic 
and magnetostatic approximations, we represent the selfelectric and self-magnetic fields as $\mathbf{E}^{s}=-\nabla \phi(\mathbf{x}, t)$ and $\mathbf{B}^{s}=\nabla \times A_{z}(\mathbf{x}, t) \hat{\mathbf{e}}_{z}$. The nonlinear Vlasov-Maxwell equations can be approximated by $[5,22]$

$$
\begin{gathered}
\left\{\frac{\partial}{\partial t}+\mathbf{v} \cdot \frac{\partial}{\partial \mathbf{x}}-\left[\gamma_{j} \mathrm{~m}_{j} \omega_{\beta j}^{2} \mathbf{x}_{\perp}\right.\right. \\
\left.+e_{j}\left(\nabla \phi-\frac{v_{z}}{c} \nabla_{\perp} \mathrm{A}_{z}\right] \cdot \frac{\partial}{\partial \mathbf{p}}\right\} f_{j}(\mathbf{x}, \mathbf{p}, t)=0,
\end{gathered}
$$

and

$$
\begin{aligned}
\nabla^{2} \phi & =-4 \pi \sum_{j} e_{j} \int d^{3} p f_{j}(\mathbf{x}, \mathbf{p}, t), \\
\nabla^{2} A_{z} & =-\frac{4 \pi}{c} \sum_{j} e_{j} \int d^{3} p v_{z} f_{j}(\mathbf{x}, \mathbf{p}, t) .
\end{aligned}
$$

In the nonlinear $\delta$ f formalism $[23,24,25]$, we divide the total distribution function into two parts, $f_{j}=f_{j 0}+\delta f_{j}$, where $f_{j 0}$ is a known equilibrium solution to the nonlinear Vlasov-Maxwell equations (1) and (2), and the numerical simulation is carried out to determine the detailed nonlinear evolution of the perturbed distribution function $\delta f_{j}$. This is accomplished by advancing the weight function defined by $w_{j} \equiv \delta f_{j} / f_{j}$, together with the particles' positions and momenta. The equations of motion for the particles, obtained from the characteristics of the nonlinear Vlasov equation (1), are given by

$$
\begin{aligned}
\frac{d \mathbf{x}_{\perp j i}}{d t} & =\left(\gamma_{j} m_{j}\right)^{-1} \mathbf{p}_{\perp j i}, \\
\frac{d z_{j i}}{d t} & =v_{z j i}=\beta_{j} c+\gamma_{j}^{-3} m_{j}^{-1}\left(p_{z j i}-\gamma_{j} m_{j} \beta_{j} c\right), \\
\frac{d \mathbf{p}_{j i}}{d t} & =-\gamma_{j} m_{j} \omega_{\beta j}^{2} \mathbf{x}_{\perp j i}-e_{j}\left(\nabla \phi-\frac{v_{z j i}}{c} \nabla_{\perp} A_{z}\right) .
\end{aligned}
$$

Here the subscript " $j i$ " labels the $i$ ' th simulation particle of the $j$ 'th species. The dynamical equations for $w_{j i}$ is $[23,25]$

$$
\begin{aligned}
\frac{d w_{j i}}{d t} & =-\left(1-w_{j i}\right) \frac{1}{f_{j 0}} \frac{\partial f_{j 0}}{\partial \mathbf{p}} \cdot \delta\left(\frac{d \mathbf{p}_{j i}}{d t}\right), \\
\delta\left(\frac{d \mathbf{p}_{j i}}{d t}\right) & \equiv-e_{j}\left(\nabla \delta \phi-\frac{v_{z j i}}{c} \nabla_{\perp} \delta A_{z}\right),
\end{aligned}
$$

where $\delta \phi=\phi-\phi_{0}$ and $\delta A_{z}=A_{z}-A_{z 0}$. Here, the equilibrium solutions $\left(\phi_{0}, A_{z 0}, f_{j 0}\right)$ solve the steady-state $(\partial / \partial t=0)$ Vlasov-Maxwell equations (1) and (2). A wide variety of axisymmetric equilibrium solutions to Eqs. (1) and (2) have been investigated in the literature. The perturbed distribution $\delta f_{j}$ is obtained through the weighted Klimontovich representation [1]

$$
\delta f_{j}=\frac{N_{j}}{N_{s j}} \sum_{i=1}^{N_{s j}} w_{j i} \delta\left(\mathbf{x}-\mathbf{x}_{j i}\right) \delta\left(\mathbf{p}-\mathbf{p}_{j i}\right),
$$

where $N_{j}$ is the total number of actual $j$ 'th species particles, and $N_{s j}$ is the total number of simulation particles for the $j$ 'th species. Maxwell's equations are also expressed in terms of the perturbed fields and perturbed density according to

$$
\nabla^{2} \delta \phi=-4 \pi \sum_{j} e_{j} \delta n_{j}, \quad \nabla^{2} \delta A_{z}=-\frac{4 \pi}{c} \sum_{j} \delta j_{z j},
$$

where

$$
\begin{aligned}
\delta n_{j} & =\frac{N_{j}}{N_{s j}} \sum_{i=1}^{N_{s j}} w_{j i} S\left(\mathbf{x}-\mathbf{x}_{j i}\right), \\
\delta j_{z j} & =\frac{e_{j} N_{j}}{N_{s j}} \sum_{i=1}^{N_{s j}} v_{z j i} w_{j i} S\left(\mathbf{x}-\mathbf{x}_{j i}\right) .
\end{aligned}
$$

Here, $S\left(\mathbf{x}-\mathbf{x}_{j i}\right)$ is a shape function distributing particles on the grids in configuration space.

The nonlinear particle simulations are carried out by iteratively advancing the particle motions, including the weights they carry, according to Eqs. (3) and (4), and updating the fields by solving the perturbed Maxwell's equations (6) with appropriate boundary conditions at the cylindrical, perfectly conducting wall. Even though it is a perturbative approach, the $\delta \mathrm{f}$ method is fully nonlinear and simulates completely the original nonlinear VlasovMaxwell equations. Compared with conventional particlein-cell simulations, the noise level in $\delta \mathrm{f}$ simulations is significantly reduced. The dominant numerical noise mechanisms in particle simulations, such as numerical collisions, are statistical. The $\delta \mathrm{f}$ method reduces the noise level of the simulations because the statistical noise, which is of order $O\left(N_{s}^{-1 / 2}\right)$ for the total distribution function in the conventional particle-in-cell (PIC) method, is only associated with the perturbed distribution function in the $\delta \mathrm{f}$ method. If the same number of simulation particles is used in the two approaches, then the noise level in the $\delta \mathrm{f}$ method is reduced by a factor of $f / \delta$ f relative to the PIC method. To achieve the same accuracy for the perturbed fields, the number of simulation particles used in the $\delta \mathrm{f}$ method is reduced by a factor of $(f / \delta f)^{2}$. For the e-p two-stream instability in the Proton Storage Ring experiment studied in this paper, we obtain satisfactory results with about $10^{5}$ simulation particles using the $\delta \mathrm{f}$ method. If the conventional PIC method is used, then for a nonlinear saturation level of $1 \%$, about $10^{4}$ times more simulation particles would be needed to achieve the same accuracy. The $\delta$ f method can also be used to study linear stability properties, provided the factor $\left(1-w_{j i}\right)$ in Eq. (4) is approximated by unity, and the forcing terms in Eq. (3) are replaced by the unperturbed force. Implementation of the 3D multispecies nonlinear $\delta \mathrm{f}$ simulation method described above is embodied in the BEST code [25] developed at the Princeton Plasma Physics Laboratory. The code advances the particle motions using a leap-frog method, and solves Maxwell's equations in cylindrical geometry. For those fast particle motions which require much larger sampling frequency $1 / \Delta t$ than the frequency of the mode being studied, the code uses an 
adiabatic field pusher to advance the particles many time steps without solving for the perturbed fields. The upper limit for $\Delta t$, the time step to advance the particles' phase space position, is normally determined by the Courant condition. For the electron-proton two-stream instability, the electrons' transverse motion requires the smallest $\Delta t$, and the mode frequency is comparable to the electron bounce frequency in the transverse direction. We can therefore update the electrons' phase space positions more often than the field. On the IBM SP-2 super computer, the BEST code advances $4.2 \times 10^{11}$ particle $\times$ time-steps when simulating the e-p two-stream instability in the Proton Storage Ring experiment.

\section{SIMULATION OF TWO-STREAM INSTABILITY}

In high-intensity accelerators and storage rings, there exists many discrete collective eigenmodes (excitations) of the ion beam. Among them, the dipole surface mode can exhibit instability when a background electron population is present [5, 12-14]. This instability is basically of the two-stream type, and is strongest when the ions are relatively cold in the propagation direction. The directed velocity difference, $V_{b}-V_{e}$, between the beam ions and the background electrons provides the free energy for the collective modes to grow. The instability observed in the Proton Storage Ring [12-14] is believed to have this twostream characteristic. We have simulated the two-stream instability using the BEST code, which self-consistently solves the nonlinear Vlasov-Maxwell equations using the $\delta f$ method. The simulation results presented here are for system parameters typical of the PSR experiment for a long coasting beam. The background distribution functions $f_{j 0}(r, \mathbf{p})$ are assumed to be the bi-Maxwellian with temperature $T_{j \perp}=$ const. in the $x-y$ plane, and temperature $T_{j \|}=$ const. in the $z$-direction, i.e.,

$$
\begin{aligned}
& f_{j 0}(r, \mathbf{p})=\frac{\hat{n}_{j}}{\left(2 \pi m_{j}\right)^{3 / 2} \gamma_{j}^{5 / 2} T_{j \perp} T_{j \|}^{1 / 2}} \\
& \times \exp \left\{-\frac{\left(p_{z}-\gamma_{j} m_{j} \beta_{j} c\right)^{2}}{2 \gamma_{j}^{3} m_{j} T_{j \|}}\right\} \times \\
& \exp \left\{-\frac{p_{\perp}^{2} / 2 \gamma_{j} m_{j}+\gamma_{j} m_{j} \omega_{\beta j}^{2} r^{2} / 2+e_{j}\left(\phi_{0}-\beta_{j} A_{z 0}\right)}{T_{j \perp}}\right\},
\end{aligned}
$$

where $\hat{n}_{j}$ is the density on axis $(r=0)$ of the $\mathrm{j}$ 'th species. $\phi_{0}$ and $A_{z 0}$ are equilibrium fields, which are coupled with Eq. (8) through the nonlinear Maxwell equations

$$
\begin{gathered}
\frac{1}{r} \frac{\partial}{\partial r} r \frac{\partial \phi_{0}(r)}{\partial r}=-4 \pi \sum_{j} e_{j} \int d^{3} p f_{j 0}(r, \mathbf{p}), \\
\frac{1}{r} \frac{\partial}{\partial r} r \frac{\partial A_{z 0}(r)}{\partial r}=-\frac{4 \pi}{c} \sum_{j} e_{j} \int d^{3} p v_{z} f_{j 0}(r, \mathbf{p}) .
\end{gathered}
$$

In the simulations, we take $\gamma_{b}=1.85, m_{e} / m_{b}=1 / 1836$, $\omega_{\beta b}=40 \mathrm{MHz}, r_{w}=5 \mathrm{~cm}, V_{e}=0$ and $\omega_{\beta e}=0$

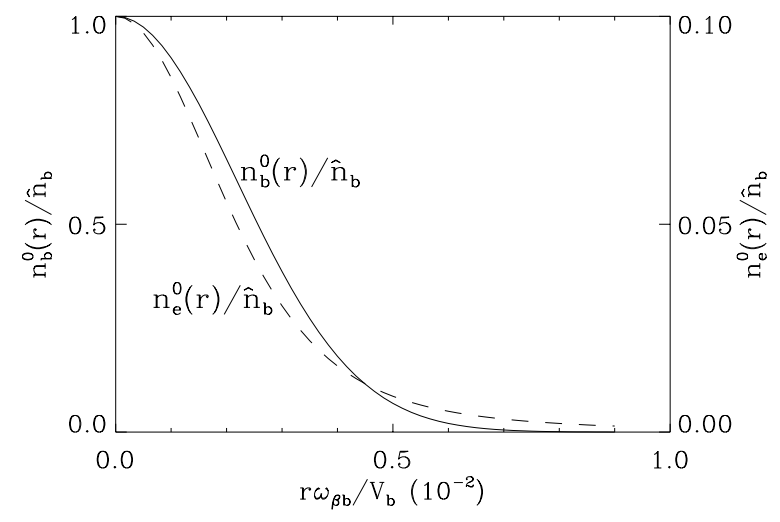

Figure 1: Plots of the equilibrium proton and electron density profiles.

(corresponding to axially stationary electrons). The spacecharge intensity varies from moderate to strong, corresponding to $0.008 \leq \hat{\omega}_{p b}^{2} / 2 \gamma_{b}^{2} \omega_{\beta b}^{2} \leq 0.158$, where $\hat{\omega}_{p b}^{2}=$ $4 \pi \hat{n}_{b} e_{b}^{2} / \gamma_{b} m_{b}$ is the ion plasma frequency-squared on axis. The fractional charge neutralization $f \equiv \hat{n}_{e} / \hat{n}_{b}$ varies from $5 \%$ to $25 \%$, where $\hat{n}_{e}$ and $\hat{n}_{b}$ are the electron and beam ion number densities on axis $(r=0)$.

Typical numerical results are illustrated in Figs. 1-3 for the "baseline" case with system parameters corresponding to $\hat{\omega}_{p b}^{2} / 2 \gamma_{b}^{2} \omega_{\beta b}^{2}=0.079, T_{b \perp} / \gamma_{b} m_{b} V_{b}^{2}=3.61 \times 10^{-6}$, $T_{e \perp} / \gamma_{b} m_{b} V_{b}^{2}=5.86 \times 10^{-7}, f=0.1, V_{e}=0$, and $\omega_{\beta e}=0$ (stationary electrons). Because the e-p instability is strongest when the beam ions are cold in the parallel direction [5] (no Landau damping by parallel kinetic effects), we take $\Delta p_{b \|}=0=\Delta p_{e \|}$ in the simulation presented in Figs. 1-3 to maximize the growth rate. Plotted in Fig. 1 is the equilibrium density profiles for the protons and electrons, $n_{j}^{0}(r)=\int d^{3} p f_{j 0}(r, \mathbf{p}, t)(j=b, e)$, which are easily obtained after the equilibrium potentials $\phi_{0}$ and $A_{z 0}$ are solved numerically from Eqs. (8) and (9). As illustrated in Fig. 2, the $x-y$ projection (at fixed value of $z$ ) of the perturbed space-charge potential $\delta \phi(x, y, t)$ grows exponentially with time during the linear phase of the instability. Clearly, the unstable mode is a dipole mode with azimuthal mode number $l=1$. It is important to emphasizes that the simulations are based on first principles - the nonlinear Vlasov-Maxwell equations. All possible mode excitations are allowed in the simulations. Simulations using typical operating parameters in the PSR experiment [12-14] indicate that the $l=1$ dipole mode is the most unstable mode. For this dominant mode in Fig. 2, the real part of the eigenfrequency is Rew $=25.13 \omega_{\beta b}$, and the normalized wavelength in the longitudinal direction is $k_{z} V_{b} / \omega_{\beta b}=26.17$. These results are in good agreement with those measured in the PSR experiments [12-14]. For the baseline case, the dependence of the instability growth rate on normalized axial wavenumber $k_{z} V_{b} / \omega_{\beta b}$ is illustrated in Fig. 3. Here, $k_{z}=2 \pi n / L=n / R$, where $R$ is the ring radius, and $n$ is the mode number. The results in Fig. 3 are qualitatively consistent with the analytical results obtained for uniform-density beams $[5,6]$. The important 

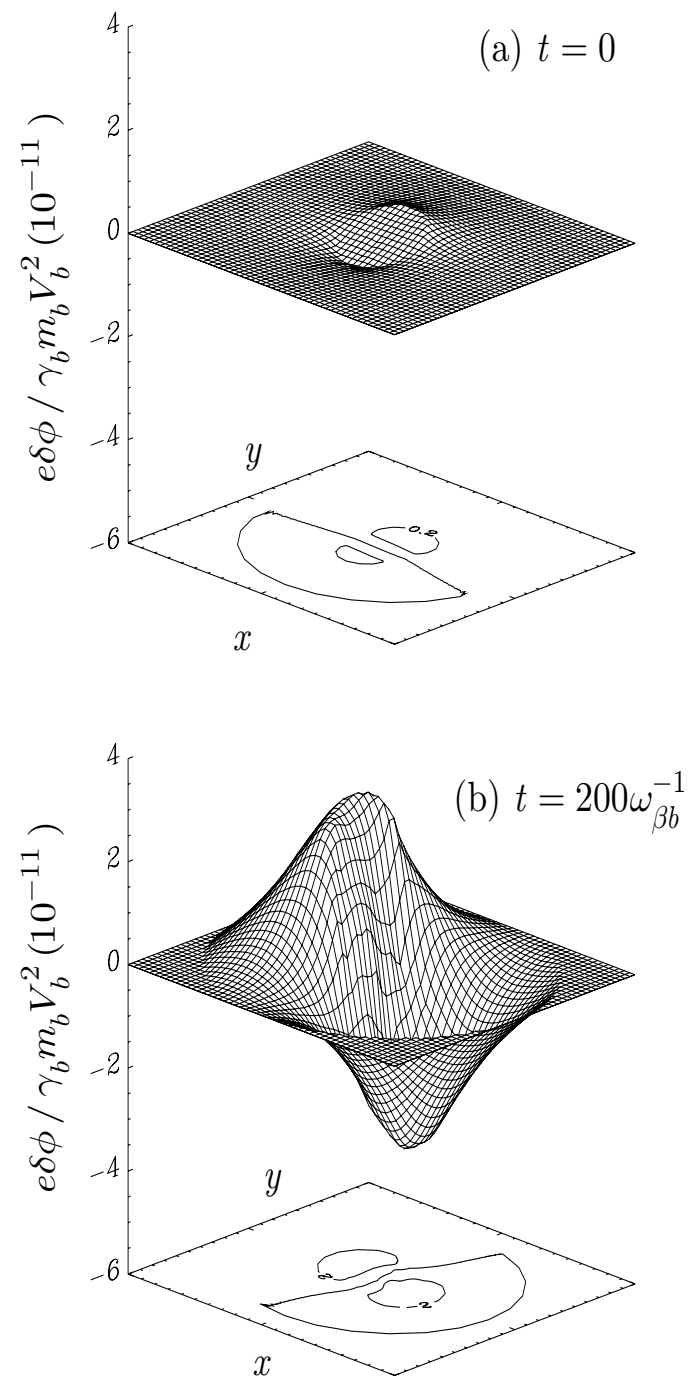

Figure 2: The $x-y$ projection (at fixed value of $z$ ) of the perturbed electrostatic potential $\delta \phi(x, y, t)$ for the electronproton two-stream instability growing from a small initial perturbation, shown at (a) $t=0$, and (b) $\omega_{\beta b} t=200$.

physics here is that only for a certain range of $k_{z} V_{b} / \omega_{\beta b}$ can the collective modes of the beam ions effectively resonate with the electrons and produce instability. In Fig. 4, for the case where $f=\hat{n}_{e} / \hat{n}_{b}=0.15$, the maximum growth rate in the simulations is plotted versus the normalized beam density $\hat{n}_{b} / \hat{n}_{b 0}$ for different values of initial axial momentum spread. Here, $\hat{n}_{b 0}=9.41 \times 10^{8} \mathrm{~cm}^{-3}$, corresponding to an average current of $35 \mathrm{~A}$ in the PSR experiment (the baseline case with $\hat{\omega}_{p b}^{2} / 2 \gamma_{b}^{2} \omega_{\beta b}^{2}=0.079$ in Figs. 1-3). It is evident from the results shown in Fig. 4 that the growth rate is an increasing function of the beam density $\hat{n}_{b} / \hat{n}_{b 0}$, but a decreasing function of the longitudinal momentum spread, which qualitatively agrees with previous analytical results [7]. A larger longitudinal momentum spread induces stronger Landau damping by parallel kinetic effects and therefore reduces the growth rate of the instability, whereas higher beam intensity provides more free energy to drive a stronger instability.

Besides the effects of longitudinal Landau damping by

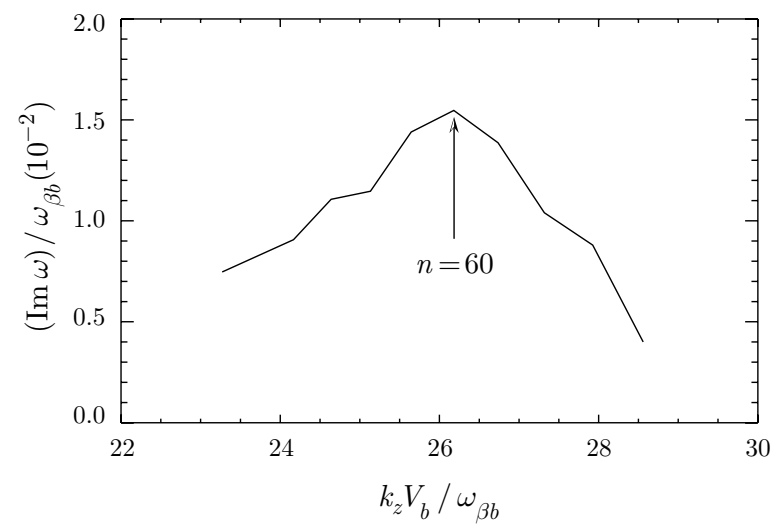

Figure 3: Plot of the normalized linear growth rate $\gamma=$ $(\operatorname{Im} \omega) / \omega_{\beta b}$ versus $k_{z} V_{b} / \omega_{\beta b}=n V_{b} / \omega_{\beta b} R$.

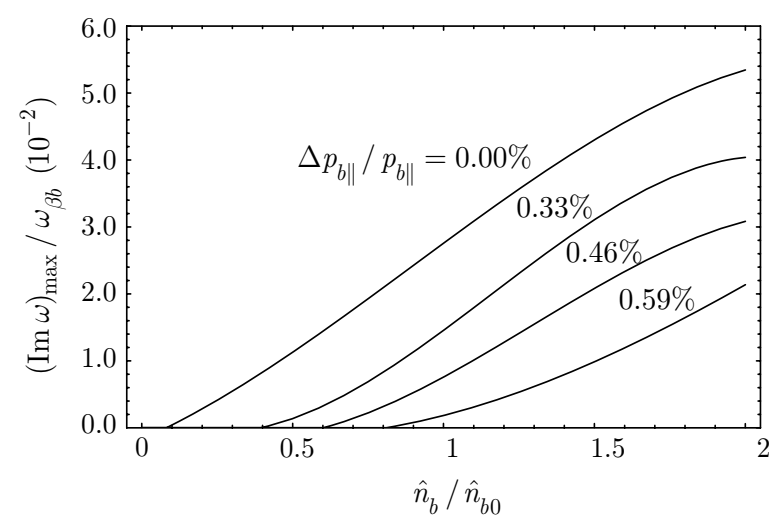

Figure 4: Maximum growth rate versus normalized beam density for different values of initail axial momentum spread of the beam ions.

the beam ions, we are also be able to simulate the stabilizing effects due to space-charge-induced tune spread, because the simulations are carried out for realistic equilibrium beams with near-Gaussian density profiles. As a result of the presence of various important damping mechanisms, an instability threshold is observed in the simulations. Plotted in Fig. 5 is the instability threshold in terms of the normalized beam density $\hat{n}_{b} / \hat{n}_{b 0}$ as a function of momentum spread $\Delta p_{b \|} / p_{b \|}$ for different vaules of fractional charge neutralization $f$. Evidently, larger momentum spread and smaller fractional charge neutralization imply a higher density threshold for the instability to occur. For a specified value of $f$, if $\left(\Delta p_{b \|} / p_{b \|}, \hat{n}_{b} / \hat{n}_{b 0}\right)$ fall bellow the curves in Fig. 5 , then there is no two-stream instability. Finally, in Fig. 6, we simulate an unstable case to its fully nonlinear phase. This case corresponds to $\hat{n}_{b} / \hat{n}_{b 0}=2$, $\hat{\omega}_{p b}^{2} / 2 \gamma_{b}^{2} \omega_{\beta b}^{2}=0.158, f=0.1$, and $\Delta p_{b \|}=0=\Delta p_{e \|}$. We see clearly from Fig. 6 the initial linear phase of the instability and the subsequent nonlinear saturation. Most important, a second phase of nonlinear growth is observed, which leads to a strong nonlinear modification of the beam density. Since the initial instability nonlinearly saturates at a relatively low level $\left(\delta n_{b} / \hat{n}_{b} \sim 0.3 \%\right)$, the late-time growth of the e-p instability observed experimentally in 


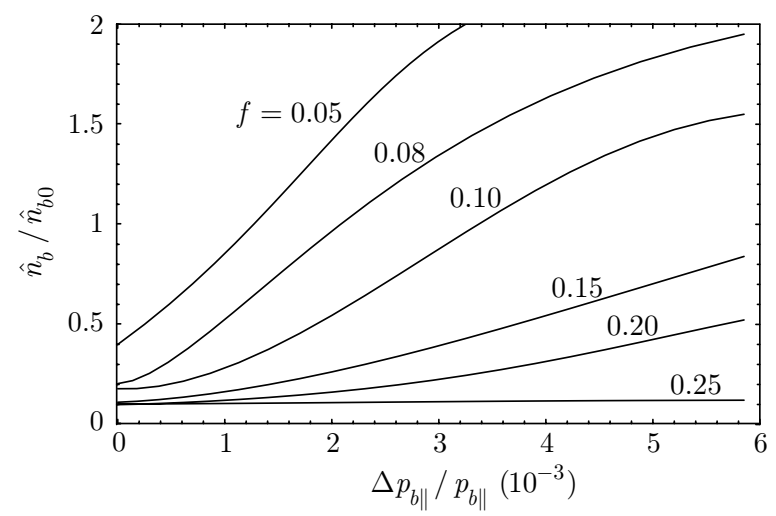

Figure 5: Density threshold for the two-stream instability as a function of beam axial momentum spread for different values of fractional charge neutralization.

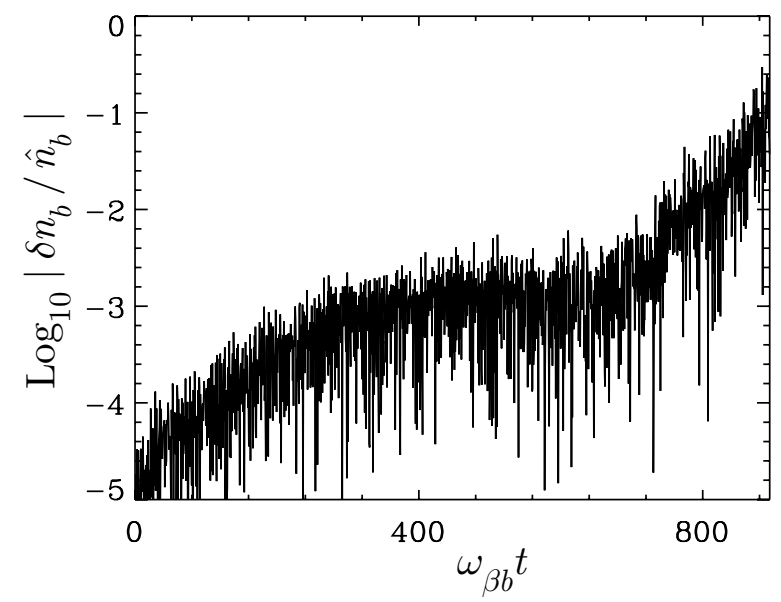

Figure 6: Linear and nonlinear phases of the e-p twostream instability.

PSR has likely passed the initial linear growth and saturation, and entered the second phase of strong nonlinear growth.

\section{CONCLUSIONS}

In conclusion, a 3D multispecies nonlinear perturbative particle simulation method has been developed to study the electron-ion two-stream instability described selfconsistently by the Vlasov-Maxwell equations. Important properties of this instability have been investigated numerically, and are found to be in qualitative agreement with theoretical predictions [5-7] and the PSR experiment [12-14]. Numerically, the instability threshold is found to decrease with increasing fractional neutralization, and increase with increasing axial momentum spread of the beam particles. In the nonlinear phase, the simulations show that the instability first saturates at a relatively low level, and subsequently grows to a higher level.

\section{REFERENCES}

[1] R. C. Davidson, Physics of Nonneutral Plasmas (AddisonWesley Publishing Co., Reading, MA, 1990), and references therein.

[2] A. W. Chao, Physics of Collective Beam Instabilities in High Energy Accelerators (Wiley, New York, 1993).

[3] T. P. Wangler, Principles of RF Linear Accelerators (John Wiley \& Sons, Inc., New York, 1998).

[4] M. Reiser, Theory and Design of Charged Particle Beams (John Wiley \& Sons, Inc., New York, 1994).

[5] R. C. Davidson, H. Qin, P. H. Stoltz, and T. -S. Wang, Phys. Rev. Special Topics on Accel. and Beams 2, 054401 (1999), and references therein.

[6] R. C. Davidson, H. Qin, and T. -S. Wang, Phys. Lett. A 252, 213 (1999).

[7] R. C. Davidson and H. Qin, Phys. Lett. A 270, 177 (1999).

[8] T.-S. Wang, P. J. Channell, R. J. Macek, R. C. Davidson, Proc. 2001 PAC.

[9] D. G. Koshkarev and P. R. Zenkevich, Particle Accel. 3, 1 (1972).

[10] E. Keil and B. Zotter, CERN Report CERN-ISR-TH/71-58 (1971).

[11] L. J. Laslett, A. M. Sessler, and D. Möhl, Nucl. Instr. Meth. A 121, 517 (1974).

[12] D. Neuffer, E. Colton, D. Fitzgerald, T. Hardek, R. Hutson, R. Macek, M. Plum, H. Thiessen, and T. -S. Wang, Nucl. Instr. Meth. Phys. Res. A321, 1 (1992).

[13] R. J. Macek, AIP Proceed. 448, 116 (1998)

[14] R. Macek, et al, Proc. 2001 PAC.

[15] M. Izawa, Y. Sato, and T. Toyomasu, Phys. Rev. Lett. 74, 5044 (1995).

[16] J. Byrd, A. Chao, S. Heifets, M. Minty, T. O. Roubenheimer, J. Seeman, G. Stupakov, J. Thomson, and F. Zimmerman, Phys. Rev. Lett. 79, 79 (1997).

[17] K. Ohmi, Phys. Rev. E 55, 7550 (1997).

[18] M. Blaskiewicz, Proc. 2000 EPAC, 1110 (2000)

[19] T. -S. Wang and L. Smith, Particle Accel. 12, 247 (1982).

[20] I. Hofmann, L. J. Laslett, L. Smith, and I. Haber, Particle Accel. 13, 145 (1983).

[21] J. Struckmeier and I. Hofmann, Particle Accel. 39, 219 (1992).

[22] R. C. Davidson and C. Chen, Particle Accel. 59,175 (1998).

[23] W. W. Lee, Q. Qian, and R. C. Davidson, Phys. Lett. A 230, 347 (1997); Q. Qian, W. W. Lee, and R. C. Davidson, Phys. Plasmas 4, 1915 (1997).

[24] P. H. Stoltz, R. C. Davidson, and W. W. Lee, Phys. Plasmas 6, 298 (1999).

[25] H. Qin, R. C. Davidson, and W. W. Lee, Phys. Rev. Special Topics on Accel. and Beams 3, 084401 (2000); 3, 109901 (2000).

[26] E. Startsev, H. Qin, R. C. Davidson, and W. Wei-li Lee, Proc. 2001 PAC (2000).

[27] R. C. Davidson, H. Qin, and P. J. Channell, Phys. Rev. Special Topics on Accel. and Beams 2, 074401 (1999); 3, 029901 (2000); P. J. Channell, Phys. Plasmas 6, 982 (1999). 